\title{
. Sexual coercion in a natural mandrill population
}

\section{Authors}

4

5 Nikolaos Smit ${ }^{1, *}$, Alice Baniel ${ }^{2,3}$, Berta Roura-Torres ${ }^{4}$, Paul Amblard-Rambert ${ }^{4}$, Marie J. E. Char-

6 pentier ${ }^{1, \alpha}$ and Elise Huchard ${ }^{1, \alpha}$

7

8 Affiliations

9

${ }_{10}{ }^{1}$ ISEM, UMR5554 - University of Montpellier/CNRS/IRD/EPHE, Place Eugène Bataillon (cc065),

1134095 Montpellier, France

${ }_{12}^{2}$ Center for Evolution and Medicine, Arizona State University, Tempe, AZ, 85281, USA

${ }_{13}^{3}$ School of Life Sciences, Arizona State University, Tempe, AZ, 85287, USA

${ }_{14}^{4}$ Projet Mandrillus, BP 52, Bakoumba, Gabon

${ }_{15}^{\alpha}$ These authors contributed equally to this work

${ }_{16}^{*}$ Correspondence to:

17 Nikolaos Smit, ORCID: 0000-0003-0440-1998

18 Phone: +33(0)782382856; email: nikos@ctemplar.com 


\section{Abstract}

Increasing evidence indicates that sexual coercion is widespread. While some coercive strategies are conspicuous, such as forced copulation or sexual harassment, less is known about the ecology and evolution of intimidation, where repeated male aggression promotes future rather than immediate mating success with targeted females. Although known in humans, intimidation was recently reported in chimpanzees and chacma baboons, where males are regularly violent against females. Here, we investigate the nature of male coercive strategies in wild mandrills (Mandrillus sphinx), a primate living in large polygynandrous groups where severe male aggression towards females is rare and females can exert mate choice. Yet, we found support for all three predictions of the sexual coercion hypothesis, namely that male aggression (1) specifically targets sexually receptive females, (2) inflicts costs to these females, and (3) increases male mating success in the long-term. These results hold true when considering only non-physical threats, or only severe aggression. Finally, we show that high-ranking females are most targeted by males, probably because of their higher reproductive performances. These results indicate that sexual intimidation is widespread in sexually dimorphic and group-living mammals, can co-occur with female mate choice, and is most costly to the most attractive females.

\section{Introduction}

The 西

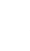
and lactation in mammals, male reproductive success is primarily determined by the number of mating partners [1]. In some species, males use sexual coercion towards females, defined as "the use by a male of force, or threat of force, that functions to increase the chances that a female will 
41

mate with him at a time when she is likely to be fertile, and to decrease the chances that she will mate with other males, at some cost to the female" [2], to improve their mating success $[2,3]$.

Behavioural ecologists have traditionally documented coercive strategies that are immediately visible, such as forced copulation (when a female is physically restrained by a male to mate with him), sexual harassment (when aggression immediately precedes copulation and is directed until the female cedes; [2]) and coercive mate-guarding (when a male aggressively herds females and enforce close proximity to prevent them to copulate with rival males; $[4,5])$. These forms of sexual coercion have been reported from insects $[6,7]$ to vertebrates $[8,9,10,11,12]$. In contrast, longterm forms of sexual coercion - when aggression does not translate immediately but subsequently into mating benefits for the aggressor - are more elusive and have been less studied outside of human societies. Sexual intimidation, when repeated male aggression aims at enforcing future female sexual compliance, has only been documented in two primate societies characterized by severe male aggression to females (chimpanzees (Pan troglodytes): [13]; chacma baboons (Papio ursinus): [14]). Similarly, males of different taxa (e.g. birds and primates including humans) can also punish females following copulations with rival males to prevent cuckoldry in the future $[15,16,17,18]$.

Sexual coercion is increasingly recognized as a driving force influencing the evolution of mating and social systems in animals [19, 2, 20], including humans [21, 22]. In mammals, male coercive tactics appear most common in polygynous and polygynandrous species, where males compete intensively over mating opportunities and a substantial fraction of males fails to secure copulations, and where sexual size dimorphism is pronounced, allowing males to threaten or harass females at low costs $[23,24]$. In these species, female impediment to male copulation attempts has been associated with an increased risk of severe injury or even death [25]. The forms of coercion used by males 
65

are then likely to vary according to the stability of male-female associations and male dominance status. Short-term strategies such as sexual harassment and forced copulations may be frequently used in solitary species, where males and females only encounter each other for mating [3]. By contrast, long-term term strategies, such as intimidation and punishment are more likely to evolve in species living in stable bisexual groups where males and females maintain medium- to long-term social relationships. Furthermore, in polygynous groups, harassment and forced copulations might be used more frequently by subordinate males that are excluded from mating opportunities [26, 27] while long-term male coercive strategies might be used more often by dominant males to constrain female promiscuity and impose closer proximity (e.g. [28]).

Primates are good candidates to study sexual coercion because the diversity of their social and mating systems may promote various male and female sexual strategies, while their extensive cognitive abilities, including individual recognition and long-term memory, may facilitate the use of long-term male coercive strategies [22]. Such strategies are also promoted by the fact that many primates live in stable bisexual groups where males and females maintain differentiated relationships, and by a widespread male-biased sexual dimorphism associated with polygynous or some polygynandrous mating systems.

In this study, we examine whether males exert sexual coercion in a large natural, polygynandrous group of mandrills (Mandrillus sphinx), an Old World primate characterized by an extreme sexual dimorphism in body size (males are 3.4 times heavier than females; [29]) and canine length [30]. Mandrills are seasonal breeders and most males immigrate in the social group at the onset of the mating season (April-September; [31]), resulting in intense male-male mating competition [32]. Male reproductive skew is high, since the alpha male monopolizes $60-70 \%$ of reproductions 
89

$[33,34]$. Female mandrills develop perineal swellings during fertility that grow in size as they approach ovulation and dominant males focus their mate-guarding efforts on maximally swollen females [35]. Yet, both sexes mate promiscuously and females exhibit some form of mate choice [36], for example by avoiding males' attempts to copulate or interrupting copulation before ejaculation (MJEC personal observation). Severe male aggression towards females occurs but appears relatively infrequent for human observers. Female relatives form tight social relationships [33], including aggressive coalitions against males that can lead to male's death (in captivity: [37]). Studying male sexual coercion in this species, where most males are temporary residents in the group during the mating season, females can retaliate against males, severe male aggression against females is inconspicuous and females display some choice over their mating partners, appears thus highly relevant.

We test the three key predictions of the sexual coercion hypothesis [2], namely that male aggression (i) targets sexually receptive females more than females in other reproductive states, (ii) is costly to females in the form of a greater exposure to injuries, and (iii) increases male mating success with the victim. For this last prediction, we further investigate different forms of coercion by testing if aggression by a male towards a female increases his chances to mate with her within the following minutes (harassment) or within a longer time-window (intimidation). We also test whether a female that has just copulated with a given male receives immediate aggression from other male(s) as a punishment. Finally, we test an alternative hypothesis to sexual coercion ("aggressive male phenotype" hypothesis) stating that the correlation between male aggression and mating is observed because females prefer to copulate with aggressive males due to direct (e.g. better infanticide protection) or indirect (i.e. better genes for their offspring; [38, 39]) fitness benefits of these male traits to females $[40,41]$. 


\section{${ }_{113}$ Methods}

\section{(a) Study system}

We studied a natural population of mandrills established in 2002 by the release of 36 captive individuals followed by the release of another 29 individuals in 2006, in the Lékédi park, a private park located in Southern Gabon [42]. Starting in 2003, wild males joined the group to reproduce. In early 2012, the Mandrillus Project was set-up to study this population, benefiting from an initial habituation of these captive-born individuals to human presence. In early 2020, only 8 adult females from ca. 210 individuals were captive-born. All individuals were individually-recognized, daily monitored and censused.

\section{(b) Female reproductive state and sex ratio}

The reproductive state of each adult female was recorded on a near-daily basis, as well as the size of sexual swelling during periods of fertility (on a scale from 0 to 3; see electronic supplementary material). Each female was classified as: "non-swollen" (i.e. non-fertile phase of the cycle that does not fall within the following three categories), "swollen" (i.e. with a perineal sexual swelling), "pregnant" (i.e. with a characteristic pregnancy swelling and/or if she gave birth 163-190 days afterwards; average gestation length: mean \pm SD: 175.0 \pm 4.7 days; [43]) or "lactating" (i.e. nursing a $\leq 6$ month-old infant without having resumed cycling). Finally, females were considered as nulliparous until their first parturition, and parous afterwards. We calculated monthly group sex ratio (SR) or group operational sex ratio (OSR) as the number of adult females (for SR) or adult females with inflating sexual swelling or swelling of maximal size (for OSR) divided by the number of subadult and adult males that were censused in the group that month (and were $\geq 9$ yrs). 


\section{(c) Behavioural data}

Trained observers, blind to the topic of this study, collected daily ad libitum behavioural observations and performed 5-min focal sampling on all study individuals [44]. In this study, we used 2182 hours of focal data collected on 81 adult females aged $\geq 4$ yrs (mean \pm SD: $26.9 \pm 39.3 \mathrm{~h}$ per female) and 670 hours collected on 34 subadult and adult males aged $\geq 9$ yrs $(19.7 \pm 29.2 \mathrm{~h}$ per male), collected from August 2012 to March 2020 (see electronic supplementary material). During focal sampling, sexual and agonistic interactions between a focal individual and its groupmates were recorded. Male aggressive events towards females included grasping/hitting $(\mathrm{n}=401)$, biting $(n=18)$, chasing $(n=65)$, lunging $(n=383)$, slapping the ground $(n=138)$ and head bobbing $(\mathrm{n}=567)$. For the analyses below, we ran the models including all these behaviours and we also replicated the analyses using only severe aggression (grasping/hitting, biting and chasing) or only threats (lunging, slapping the ground and head bobbing) because both categories produce different female behavioural reactions (see discussion). Dominance ranks were calculated separately for each sex using the outcomes of approach-avoidance interactions on a yearly basis (for females) or on a monthly basis (for males; see electronic supplementary material).

\section{(d) Injuries}

We recorded the occurrence, type of wound, freshness and body location of any injury on a neardaily basis on all subjects [45]. A total of 90 injuries (limping $n=15$, puncture of the skin $n=11$, bleeding or swollen skin $n=48$, other $n=16$ ) were recorded on 43 adult females over the study period. For most injuries, we did not witness the interaction and the cause but in the three cases with a known context the injury was inflicted by an adult male. 


\section{(e) Statistical Analyses}

To test whether male aggression targets swollen females preferentially (first prediction), we ran a binomial GLMM with a logit link function to study the relationship between the probability that a female received aggression by any adult male during that female focal observation (0/1; response variable) and her reproductive state at the time of observation (non-swollen, swollen, pregnant and lactating; for sample sizes, see table S1). We further controlled for the following fixed effects: female dominance rank (high-, medium- or low-ranking), parity (nulliparous or parous) and SR. The duration of focal observation $(\leq 5 \mathrm{~min})$ was log-transformed and fitted as an offset variable. Female identity and the year of focal observation were fitted as random factors. Second, we ran a similar model (same structure of fixed and random effects) with the response variable corresponding to the probability that a female received aggression by groupmates other than adult males. By doing so, we tested if swollen females were generally more targeted than any other female, regardless of the age-sex group of the aggressor.

To test whether swollen females were more injured than females in other states (second prediction), we ran a binomial GLMM with a logit link function to study the relationship between the probability that a female got injured on a given day $(0 / 1$; response variable) and her reproductive state that same day. As above, we further controlled for the following variables: female dominance rank and parity, and SR. Female identity and the year of focal observation were fitted as random factors (table S1).

We then tested whether males who were more aggressive also benefited from higher mating success with their victim (third prediction). To study intimidation, we performed a binomial GLMM with 
a logit link function to test whether the rate of aggression received by a female from a given male (continuous fixed effect) before the next estrous cycle of the female increased the probability of copulation of that heterosexual dyad during the female's swollen period ( $0 / 1$; response variable). The "aggression window" before the swollen period was defined as the time elapsed between the onset of the mating season (for resident males; see electronic supplementary material) or a male's arrival in the group a given year (for immigrant males) and until the beginning of the swollen period of the female (spanning from the first day of a female's sexual swelling to the last day where swelling size was maximal: mean \pm SD: 10.6 \pm 5.1 days; figure $\mathrm{S} 1)$. We pooled focal observations from adult females and males (table S1). We controlled for the following fixed effects in our model: female dominance rank and parity, OSR in the month corresponding to the first day of maximal swelling and male dominance rank (alpha or non-alpha) that same month in interaction with the rate of male aggression (to test whether the aggression of alpha males had a greater impact on their mating success than the aggression of subordinate males). Female identity, male identity and year of observation were fitted as random factors. The total focal observation time of the studied heterosexual dyads (during the swollen period) was log-transformed and fitted as an offset variable. We restricted our analyses to those heterosexual dyads that were observed at least for 30 minutes of focal time during the studied period (aggression window and swollen period) to avoid biases due to under-sampling. We further ran the same model but restricting the swollen period to the few days of the cycle during which the female was maximally swollen (i.e. where the probability of conception is the highest; mean \pm SD: $2.9 \pm 2.9$ days). To test for immediate effects of male aggression, we ran the same model as above considering the rate of aggression received by a female from a given male during her swollen period only (figure S1, top line). 
aggressive event recorded from a male to a swollen female whether a copulation occurred or not between that same heterosexual dyad in the next 150 seconds following the aggression (see electronic supplementary material; figure S2a). To test for male punishment, we assessed for each female and male focal observation with a copulation event recorded between a male and a swollen female whether an aggression from a different male occurred towards the copulating female in the 150 following seconds (figure S2b; table S1).

We explored an alternative scenario to sexual coercion, the "aggressive male phenotype" hypothesis, to test whether males with aggressive phenotypes have higher mating success than less aggressive males, solely because aggression may act as a sexual trait chosen by females. We reran the GLMM used for testing the occurrence of intimidation, including as an explanatory variable the overall rate of aggression directed towards any groupmate (except for adult females) during the corresponding mating season.

We ran all the above statistical tests in $\mathrm{R}$ version 4.0.3. For generalized linear mixed models (GLMMs; summarized in table S1) we used the glmer function of the lme4 package ([46]; see electronic supplementary material for technical details).

\section{Results}

\section{(a) Prediction 1: Male aggression targets swollen females}

Swollen females received significantly more aggression from adult males (mean \pm SD: 0.613 \pm 1.070 bouts per hour) than females in any other reproductive state (non-swollen: $0.331 \pm 0.661$, pregnant: 0.309 \pm 0.528 and lactating: $0.288 \pm 0.562$; figure 1a, table 1). Such pattern was found for both severe 
aggression (rate towards swollen females: $0.349 \pm 0.948$ bouts/hour, Chisq=12.539, p-value=0.006) and threats $(0.260 \pm 0.390$ bouts/hour, Chisq $=8.660$, p-value $=0.034)$. By contrast, swollen females were not more targeted by other groupmates (figure S3, table S2). In addition, high-ranking females received more male aggression than lower-ranking females (high-ranking females: $0.461 \pm 0.328$ bouts/hour, medium-ranking females: $0.216 \pm 0.240$, low-ranking females: $0.148 \pm 0.149$, table 1 ).

\section{(b) Prediction 2: Swollen females are more injured}

Swollen females were, on average, about five times more likely to become injured (mean \pm SD: $0.005 \pm 0.016$ injuries per day) than females in any other reproductive state (non-swollen: $0.001 \pm 0.004$ pregnant: $0.001 \pm 0.002$ and lactating: 0.001 \pm 0.002 ; figure 1b). None of the other fixed factors, including female rank, parity and the group sex-ratio were significantly correlated with the probability of injury (table 2).

\section{(c) Prediction 3: Aggressive males have higher mating success with their victim}

We found support for sexual intimidation in mandrills: the rate of male aggression received by a female during the time window preceding her swollen period (starting at the onset of a given mating season for resident males or a male's arrival in the group a given year for immigrant males) positively influenced the probability of copulation of the dyad during that swollen period (figure 1c, table 3). Namely, in dyads that did copulate, the rate of male-to-female aggression before the swollen period was $0.083 \pm 0.419($ mean \pm SD) times per hour, while in dyads that did not copulate, this rate fell to $0.030 \pm 0.110$. Alpha males copulated more than subordinate males, while female rank, parity, OSR and the interaction between male rank and aggression (Chisq=0.030, p-value $=0.862$ ) were not significantly correlated with the probability of copulation (table 3 ). The correlation between male aggression and mating within dyads remained significant when restricting 
the swollen period to the few days where a female was maximally swollen (i.e. close to ovulation, Chisq $=4.574, \mathrm{p}$-value $=0.032$ ). However, the rate of male aggression calculated during the swollen period of the female (instead of before) did not significantly predicted the probability of copulation during that same swollen period (table S3a). This indicates that immediate aggression (i.e. during the swollen period) did not strongly influence female mating pattern, while previous aggressive interactions over a longer period (i.e. before the swollen period) did. The pattern of correlation between aggression and subsequent mating holds when only including severe aggression (table S3b) and become marginally non-significant when only including threats (table S3c), while the rate of severe aggression and the rate of threats a female receives from a male were moderately correlated (Kendall's tau $=0.28, \mathrm{p}$-value $<10^{-3}$ ). Lastly, we failed to find evidence for a female preference for aggressive male phenotypes, as females were not more likely to mate with the most aggressive males in the group (see electronic supplementary material).

We did not find support for sexual harassment and punishment. Females copulated immediately (i.e. within 150 seconds) after aggression with their aggressor in only three out of 38 total cases of aggression observed between a male and a swollen female. Similarly, males were never observed directing aggression to a female in the 150 seconds after she copulated with a rival male (out of 173 observed copulations). Those sample sizes precluded any further formal testing of those hypotheses.

\section{Discussion}

We found support for all three core predictions of the sexual coercion hypothesis in mandrills. First, swollen females received significantly more male aggression than other females. Elevated 
aggression towards females around ovulation has been observed frequently in mammals, even in species where females dominate males socially (e.g. spotted hyena (Crocuta crocuta): [47]), suggesting that sexual coercion is widespread. Second, swollen female mandrills were significantly more injured than females in other reproductive states. Such injuries are most likely caused by males because aggression from other groupmates did not intensify during female sexually receptivity. Male aggression thus potentially causes important fitness costs in female mandrills, as shown in other mammals exhibiting sexual coercion (e.g. feral sheep (Ovis aries): [48]; bottlenose dolphins (Tursiops cf. aduncus): [49], chacma baboons: [14], chimpanzees: [50]). These fitness costs may push females to comply and copulate more with aggressive males to avoid conflict escalation and the associated risk of injury $[51,52]$. Third, we showed that increased and repeated male aggression before the receptive period improves male mating success with the targeted female at times where she is most likely fertile. This correlation holds true both with severe aggression and non-physical threats, which are only moderately correlated. Most studies on sexual coercion have focused exclusively on severe aggression $[13,14]$ but our results indicate that male mandrills use a wide aggressive repertoire, including threats, to coerce females. In this species, male threats (such as head-bob or ground-slap) typically produce little immediate behavioural reactions in females, but could increase their sexual compliance with the aggressor when exerted repeatedly [28], especially when male-female power asymmetry is high, like in mandrills which display one of the largest sexual dimorphism in primates.

The observed correlation between male aggression and mating success does not seem well-explained by alternative interpretations to sexual coercion, as we failed to find evidence supporting a female preference for particularly aggressive males. Females could potentially use male aggression as a badge of status $[13,53]$ to infer male competitive abilities, which may provide females with direct 
or indirect benefits $[40,41]$. However, in our data, variation in aggression rates among heterosexual dyads explain male mating success better than male general aggressiveness, suggesting that male mating success reflects relational properties more than male aggressive phenotype.

Our analyses reveal important aspects of the ecology of sexual coercion in mandrill societies. While we failed to find evidence for sexual harassment, repeated aggression over extended periods increases female propensity to accept mating attempts from their aggressors once they become fertile, and may further encourage them to stay around males who mate-guard them, as observed in hamadryas baboons (Papio hamadryas; [28]). Sexual intimidation has previously been shown in chimpanzees and chacma baboons $[13,14]$, two species characterized by relatively high male violence towards females. We found that male mandrills use severe aggression towards swollen females more often than chacma baboons (mean \pm SD: $0.350 \pm 0.950$ vs $0.130 \pm 0.190$ times per hr; [14]) and at a rate that lies high within the chimpanzee's reported range [13, 50]. Such frequent use of coercion by mandrill males may relate to the fact that - unlike chimpanzees and chacma baboons - they breed seasonally, thus have a limited time window to achieve matings. Yet, swollen female mandrills are injured ca. three times less than chacma baboons (mean \pm SD: $0.005 \pm 0.016$ vs $0.014 \pm 0.022$ injuries per day; [14]). Hence, although male to female aggression is more frequent in mandrills than in chacma baboons, violent aggression resulting in serious injuries is probably less common.

Moreover, the fact that we did not find any evidence of punishment, likely reflects the absence of exclusive mating bonds in mandrills (outside mate-guarding episodes) and the ability of females to sneakily escape male monopolization strategies in their dense habitat. Punishment by males in response to female sexual activity with a rival has, for instance, been reported in geladas (Thero- 
pithecus gelada) which live in more open habitat [17] and where one leader male can aggressively defend sexual access to females from his family unit [54]. To sum-up, our results are generally consistent with expectations based on the socio-ecology of mandrills, who (i) are highly dimorphic thus where males pay low costs of inter-sexual aggression, (ii) breed seasonally, and where males face high pressure to mate in a relatively short period, and (iii) live in a polygynandrous mating system, and where males and females form differentiated social bonds - allowing intimidation to function - but no exclusive mating bonds, preventing the use of punishment by males.

Our analyses further highlight that all females are not equally targeted by males. High-ranking females specifically receive more male aggression than low-ranking females, which may reflect male mating preferences because dominant females show better reproductive performances than subordinates [55, 43]. Similarly, male hyenas mate preferentially with high-ranking females $[56,57]$ while male chimpanzees direct more aggression towards parous than nulliparous females [13] and prefer old females [58], who have a higher rank and reproductive success than younger ones [59]. This result indicates that the highest costs of coercion are born by the most attractive females, as found in chimpanzees [13] and humans [60].

An important question remains whether and how female mandrills may navigate such a coercive landscape while still expressing some mate choice [32]. Chimpanzee studies have raised contrasting results, with sexual coercion in some populations $[13,50]$ versus female mate choice in other populations $[61,62]$. While differences across populations may explain these divergent findings, our work indicates that sexual coercion can co-occur with female mate choice, as reported in humans and some other species $[62,63,64,65]$. Several mechanisms may help females to mitigate the constraints set by male coercion on their own reproductive strategies. They may form alliances 
with other females to defend themselves $[3,66]$ or heterosexual bonds with males who protect them [67]. They may also appease male aggressors to limit the risk of escalation and injuries [28, 52], fight-back against aggressors, flee, hide or close their genitals [68, 69]. Female mandrills may use some of these strategies, as their behavioural repertoire includes avoiding male approaches, laying down when males attempt to copulate with them, refusing some mating attempts [32, 36], interrupting copulation by fleeing away, seeking support from subordinate males against dominant ones (MJEC personal observation) or even forming violent coalitions against high-ranking males ([37], NS personal observation). In addition, previous studies on primates have demonstrated that female reproductive synchrony and large group sizes limit female monopolization by males (across species: [70]; in mandrills: [34]) and increase the potential for females to express their strategies, including mate choice or promiscuity [71, 72]. Therefore, the extreme size of mandrill social groups along with female reproductive synchrony, may facilitate the expression of female reproductive strategies and reduce male coercion.

Here we report new evidence for sexual intimidation in a species where males, despite being much larger than females, are not conspicuously aggressive towards them (at least from a human observer perspective). The temporal uncoupling between male aggression and copulation explains why sexual intimidation may have long been overlooked, while it increasingly appears influential at shaping the social structure and mating system of polygynandrous mammals. Our results further add to a growing body of evidence that underlines the possible coexistence of male coercion and female mate choice. 
Ethics: All applicable international, national, and/or institutional guidelines for the care and use of animals were followed. This study was approved by the CENAREST institute (permit number, AR003/20/MESRSTT/CENAREST/CG/CST/CSAR) and adhered to the legal requirements of Gabon for the ethical treatment of non-human primates.

Data accessibility: The datasets and scripts necessary to replicate analyses included in this paper are deposited in the public depository: https://gitlab.com/nksmt/mandrills

Authors' contributions: N.S., M.J.E.C., and E.H. designed the study; B.R.T. and P.A.R collected behavioural data; N.S. performed the statistical analyses; N.S., M.J.E.C., E.H. wrote the original draft and all authors critically contributed to the draft and approved submission.

Competing interests: The authors declare no competing interests.

Funding: Several grants have funded the long-term collection of the data used in this study: Deutsche Forschungsgemeinschaft (DFG, KA 1082-20-1), SEEG Lékédi (INEE-CNRS) and Agence Nationale de la Recherche (ANR SLEEP 17-CE02-0002) to M.J.E.C, and ANR ERS-17-CE02-0008 to E.H. N.S. was funded by the State Scholarships Foundation (IKY) under the scholarship program from the proceeds of the "Nic. D. Chrysovergis" bequest.

Acknowledgements: The present work would not have been possible without the work of past and present field assistants of the Mandrillus Project and the logistical support of SODEPALCOMILOG society. This is a Project Mandrillus publication $n^{\mathrm{o}} \mathrm{xx}$ and ISEM $n^{\mathrm{o}} \mathrm{xx}$. 


\section{References}

[1] Bateman AJ. 1948 Intra-Sexual Selection in Drosophila. Heredity 2, 349-368.

[2] Smuts BB, w. Smuts R. 1993 Male Aggression and Sexual Coercion of Females in Nonhuman Primates and Other Mammals: Evidence and Theoretical Implications. In Advances in the Study of Behavior vol. 22 pp. 1-63. Elsevier.

[3] Clutton-Brock T, Parker G. 1995 Sexual Coercion in Animal Societies. Animal Behaviour 49, 1345-1365.

[4] Muller MN, Thompson ME, Kahlenberg SM, Wrangham RW. 2011 Sexual Coercion by Male Chimpanzees Shows That Female Choice May Be More Apparent than Real. Behavioral Ecology and Sociobiology 65, 921-933.

[5] King SL, Allen SJ, Krützen M, Connor RC. 2019 Vocal Behaviour of Allied Male Dolphins during Cooperative Mate Guarding. Animal Cognition 22, 991-1000.

[6] Arnqvist G. 1989 Multiple Mating in a Water Strider: Mutual Benefits or Intersexual Conflict?. Animal Behaviour 38, 749-756.

[7] Parker G. 1979 SEXUAL SELECTION AND SEXUAL CONFLICT. In Sexual Selection and Reproductive Competition in Insects pp. 123-166. Elsevier.

[8] Head ML, Brooks R. 2006 Sexual Coercion and the Opportunity for Sexual Selection in Guppies. Animal behaviour 71, 515-522.

[9] Bro-Jørgensen J. 2011 Intra-and Intersexual Conflicts and Cooperation in the Evolution of Mating Strategies: Lessons Learnt from Ungulates. Evolutionary Biology 38, 28-41. 
[10] McKinney F, Evarts S. 1998 Sexual Coercion in Waterfowl and Other Birds. Ornithological Monographs pp. 163-195.

[11] Galdikas BMF. 1985 Subadult Male Orangutan Sociality and Reproductive Behavior at Tanjung Puting. American Journal of Primatology 8, 87-99.

[12] Connor RC, Vollmer N. 2009 Sexual Coercion in Dolphin Consortships: A Comparison with Chimpanzees. Sexual coercion in primates: An evolutionary perspective on male aggression against females pp. 218-243.

[13] Muller MN, Kahlenberg SM, Emery Thompson M, Wrangham RW. 2007 Male Coercion and the Costs of Promiscuous Mating for Female Chimpanzees. Proceedings of the Royal Society B: Biological Sciences 274, 1009-1014.

[14] Baniel A, Cowlishaw G, Huchard E. 2017 Male Violence and Sexual Intimidation in a Wild Primate Society. Current Biology 27, 2163-2168.e3.

[15] Clutton-Brock TH, Parker GA. 1995 Punishment in Animal Societies. Nature 373, 209-216.

[16] Valera F, Hoi H, Krištín A. 2003 Male Shrikes Punish Unfaithful Females. Behavioral Ecology 14, 403-408.

[17] le Roux A, Snyder-Mackler N, Roberts EK, Beehner JC, Bergman TJ. 2013 Evidence for Tactical Concealment in a Wild Primate. Nature Communications 4, 1462.

[18] Rodseth L, Novak SA. 2009 The Political Significance of Gender Violence. Sexual coercion in primates and humans pp. 292-321.

[19] Thornhill R, Alcock J et al.. 1983 The Evolution of Insect Mating Systems. Harvard University Press. 
426

427

428

429

430

431

432

433

434

435

436

437

438

439

440

441

442

443

444

[20] Clutton-Brock T. 2021 Social Evolution in Mammals. Science 373, eabc9699.

[21] Smuts B. 1992 Male Aggression against Women: An Evolutionary Perspective. Human Nature $3,1-44$.

[22] Stumpf RM, Martinez-Mota R, Milich KM, Righini N, Shattuck MR. 2011 Sexual Conflict in Primates. Evolutionary Anthropology: Issues, News, and Reviews 20, 62-75.

[23] Nunn C. 2000 Social Evolution in Primates: The Relative Roles of Ecology and Intersexual Conflict. Infanticide by Males pp. 388-419.

[24] Cassini MH. 2021 Sexual Aggression in Mammals. Mammal Review 51, 247-255.

[25] Cassini M. 2000 A Model on Female Breeding Dispersion and the Reproductive Systems of Pinnipeds. Behavioural Processes 51, 93-99.

[26] Boeuf BJL, Mesnick S. 1991 Sexual Behavior of Male Northern Elephant Seals: I. Lethal Injuries to Adult Females. Behaviour 116, 143-162.

[27] Kunz JA, Duvot GJ, Willems EP, Stickelberger J, Spillmann B, Utami Atmoko SS, van Noordwijk MA, van Schaik CP. 2021 The Context of Sexual Coercion in Orang-Utans: When Do Male and Female Mating Interests Collide?. Animal Behaviour 182, 67-90.

[28] Swedell L, Schreier A. 2009 Male Aggression towards Females in Hamadryas Baboons: Conditioning, Coercion, and Control. Sexual coercion in primates and humans: an evolutionary perspective on male aggression against females. Harvard University Press, Cambridge pp. $244-268$. 
[29] Setchell JM, Lee PC, Wickings EJ, Dixson AF. 2001 Growth and Ontogeny of Sexual Size Dimorphism in the Mandrill (Mandrillus Sphinx). American Journal of Physical Anthropology 115, 349-360.

[30] Leigh SR, Setchell JM, Charpentier M, Knapp LA, Wickings EJ. 2008 Canine Tooth Size and Fitness in Male Mandrills (Mandrillus Sphinx). Journal of Human Evolution 55, 75-85.

[31] Brockmeyer T, Kappeler PM, Willaume E, Benoit L, Mboumba S, Charpentier MJ. 2015 Social Organization and Space Use of a Wild Mandrill ( Mandrillus Sphinx ) Group: Mandrill Social Organization and Space Use. American Journal of Primatology 77, 1036-1048.

[32] Setchell JM. 2016 Sexual Selection and the Differences between the Sexes in Mandrills ( $M$ Andrillus Sphinx ): SEXUAL SELECTION IN MANDRILLUS SPHINX. American Journal of Physical Anthropology 159, 105-129.

[33] Charpentier MJE, Harté M, Poirotte C, de Bellefon JM, Laubi B, Kappeler PM, Renoult JP. 2020 Same Father, Same Face: Deep Learning Reveals Selection for Signaling Kinship in a Wild Primate. Science Advances 6, eaba3274.

[34] Charpentier M, Peignot P, Hossaert-McKey M, Gimenez O, Setchell JM, Wickings EJ. 2005 Constraints on Control: Factors Influencing Reproductive Success in Male Mandrills (Mandrillus Sphinx). Behavioral Ecology 16, 614-623.

[35] Setchell JM, Charpentier M, Wickings EJ. 2005 Mate Guarding and Paternity in Mandrills: Factors Influencing Alpha Male Monopoly. Animal Behaviour 70, 1105-1120.

[36] Setchell JM. 2005 Do Female Mandrills Prefer Brightly Colored Males?. International Journal of Primatology 26, 715-735. 
[37] Setchell JM, Knapp LA, Wickings EJ. 2006 Violent Coalitionary Attack by Female Mandrills against an Injured Alpha Male. American Journal of Primatology 68, 411-418.

[38] Fisher RA. 1915 The Evolution of Sexual Preference. The Eugenics Review 7, 184-192.

[39] Huk T, Winkel W. 2007 Testing the Sexy Son Hypothesis-a Research Framework for Empirical Approaches. Behavioral Ecology 19, 456-461.

[40] Cordero C, Eberhard WG. 2003 Female Choice of Sexually Antagonistic Male Adaptations: A Critical Review of Some Current Research. J. EVOL. BIOL. p. 6.

[41] Pizzari T, Snook RR. 2003 Perspective: Sexual Conflict and Sexual Selection: Chasing Away Paradigm Shifts. Evolution 57, 1223-1236.

[42] Peignot P, Charpentier MJ, Bout N, Bourry O, Massima U, Dosimont O, Terramorsi R, Wickings EJ. 2008 Learning from the First Release Project of Captive-Bred Mandrills Mandrillus Sphinx in Gabon. Oryx 42.

[43] Dezeure J. 2022 Fitness Effects of Seasonal Birth Timing in a Long-Lived Social Primate Living in the Equatorial Forest. Animal Behaviour p. 17.

[44] Altmann J. 1974 Observational Study of Behavior: Sampling Methods. Behaviour 49, 227267.

[45] Dibakou SE, Basset D, Souza A, Charpentier M, Huchard E. 2019 Determinants of Variations in Fecal Neopterin in Free-Ranging Mandrills. Frontiers in Ecology and Evolution 7, 368.

[46] Bates D, Maechler M, Bolker [aut B, cre, Walker S, Christensen RHB, Singmann H, Dai B, Scheipl F, Grothendieck G, Green P, Fox J, Bauer A, copyright on simulate.formula) PNKs. 2020 Lme4: Linear Mixed-Effects Models Using 'Eigen' and S4. . 
[47] Szykman M, Engh AL, Horn RCV, Boydston EE, Scribner KT, Holekamp KE. 2003 Rare Male Aggression Directed toward Females in a Female-Dominated Society: Baiting Behavior in the Spotted Hyena. p. 18.

[48] Réale D, Boussès P, Chapuis JL. 1996 Female-Biased Mortality Induced by Male Sexual Harassment in a Feral Sheep Population. Canadian Journal of Zoology 74, 1812-1818.

[49] Wallen MM, Patterson EM, Krzyszczyk E, Mann J. 2016 The Ecological Costs to Females in a System with Allied Sexual Coercion. Animal Behaviour 115, 227-236.

[50] Watts DP. 2022 Male Chimpanzee Sexual Coercion and Mating Success at Ngogo. American Journal of Primatology n/a, e23361.

[51] Cassini MH. 2020 A Mixed Model of the Evolution of Polygyny and Sexual Size Dimorphism in Mammals. Mammal Review 50, 112-120.

[52] Baniel A, Webb CE, Cowlishaw G, Huchard E. 2021 The Submissive Pattern of Postconflict Affiliation in Asymmetric Relationships: A Test in Male and Sexually Coerced Female Baboons. Animal Behaviour 175, 87-97.

[53] Setchell JM, Jean Wickings E. 2005 Dominance, Status Signals and Coloration in Male Mandrills (Mandrillus Sphinx). Ethology 111, 25-50.

[54] Snyder-Mackler N, Alberts SC, Bergman TJ. 2012 Concessions of an Alpha Male? Cooperative Defence and Shared Reproduction in Multi-Male Primate Groups. Proceedings of the Royal Society B: Biological Sciences 279, 3788-3795.

[55] Setchell JM, Lee PC, Wickings EJ, Dixson AF. 2002 Reproductive Parameters and Maternal Investment in Mandrills (Mandrillus Sphinx). International Journal of Primatology p. 18. 
[56] Szykman M, Engh AL, Van Horn RC, Funk SM, Scribner KT, Holekamp KE. 2001 Association Patterns among Male and Female Spotted Hyenas (Crocuta Crocuta) Reflect Male Mate Choice. Behavioral Ecology and Sociobiology 50, 231-238.

[57] Keddy-Hector AC. 1992 Mate Choice in Non-Human Primates. American Zoologist 32, 62-70.

[58] Muller MN, Thompson ME, Wrangham RW. 2006 Male Chimpanzees Prefer Mating with Old Females. Current Biology 16, 2234-2238.

[59] Pusey A, Williams J, Goodall J. 1997 The Influence of Dominance Rank on the Reproductive Success of Female Chimpanzees. Science 277, 828-831.

[60] Felson RB, Cundiff PR. 2014 Sexual Assault as a Crime Against Young People. Archives of Sexual Behavior 43, 273-284.

[61] Stumpf RM, Boesch C. 2006 The Efficacy of Female Choice in Chimpanzees of the Taï Forest, Côte d'Ivoire. Behavioral Ecology and Sociobiology 60, 749-765.

[62] Kaburu SSK, Newton-Fisher NE. 2015 Trading or Coercion? Variation in Male Mating Strategies between Two Communities of East African Chimpanzees. Behavioral Ecology and Sociobiology 69, 1039-1052.

[63] Buss DM. 2007 The Evolution of Human Mating. Acta Psychologica Sinica 39, 502-512.

[64] Knott CD, Emery Thompson M, Stumpf RM, McIntyre MH. 2010 Female Reproductive Strategies in Orangutans, Evidence for Female Choice and Counterstrategies to Infanticide in a Species with Frequent Sexual Coercion. Proceedings of the Royal Society B: Biological Sciences 277, 105-113. 
[65] Bisazza A, Vaccari G, Pilastro A. 2001 Female Mate Choice in a Mating System Dominated by Male Sexual Coercion. Behavioral Ecology 12, 59-64.

[66] Paoli T. 2009 The Absence of Sexual Coercion in Bonobos. Sexual coercion in primates and humans pp. 410-423.

[67] Smuts B. 1995 The Evolutionary Origins of Patriarchy. Human Nature 6, 1-32.

[68] Eberle M, Kappeler PM. 2004 Selected Polyandry: Female Choice and Inter-Sexual Conflict in a Small Nocturnal Solitary Primate (Microcebus Murinus). Behavioral Ecology and Sociobiology 57, 91-100.

[69] Huchard E, Canale CI, Le Gros C, Perret M, Henry PY, Kappeler PM. 2012 Convenience Polyandry or Convenience Polygyny? Costly Sex under Female Control in a Promiscuous Primate. Proceedings of the Royal Society B: Biological Sciences 279, 1371-1379.

[70] Ostner J, Nunn CL, Schülke O. 2008 Female Reproductive Synchrony Predicts Skewed Paternity across Primates. Behavioral Ecology 19, 1150-1158.

[71] Ims RA. 1990 The Ecology and Evolution of Reproductive Synchrony. Trends in Ecology 86 Evolution 5, 135-140.

[72] Fürtbauer I, Mundry R, Heistermann M, Schülke O, Ostner J. 2011 You Mate, I Mate: Macaque Females Synchronize Sex Not Cycles. PLoS ONE 6, e26144. 

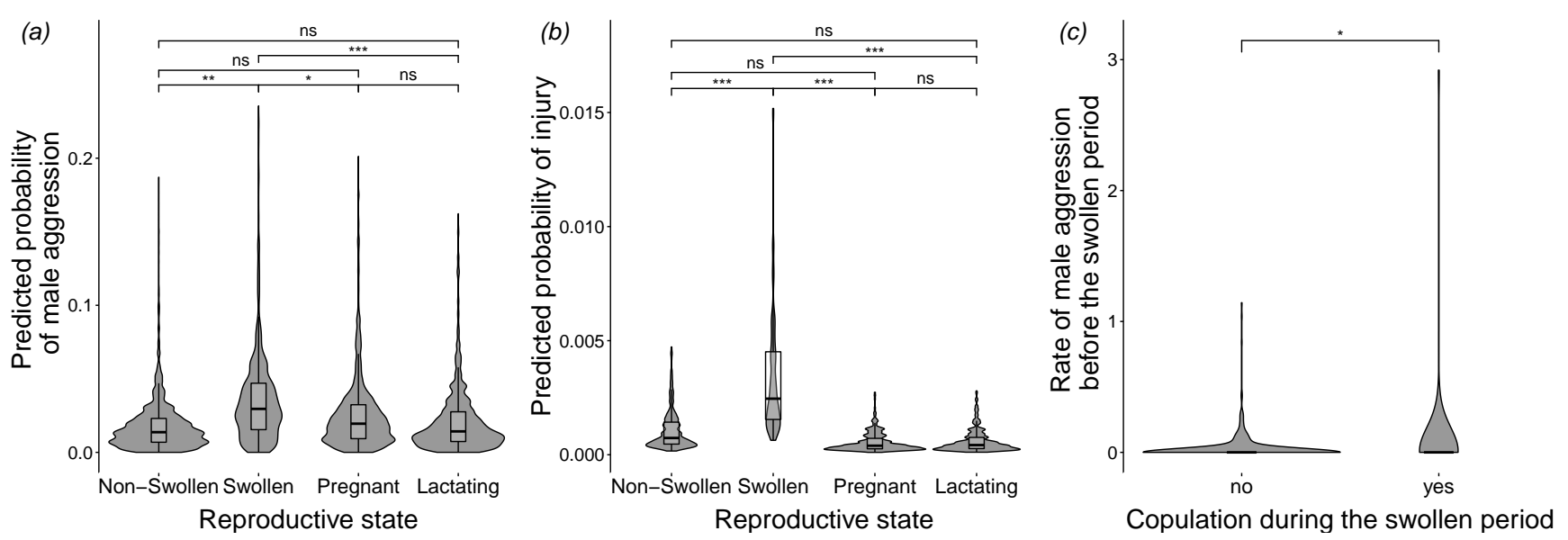

Figure 1: Results of the tests of the three predictions of the sexual coercion hypothesis. (a) Predicted probability of male aggression received by adult females as a function of their reproductive state. (b) Predicted probability for females to be injured as a function of their reproductive state. (c) Rates of male aggression (number of events per hour) received by adult females before their swollen period for heterosexual dyads who mated versus dyads that did not mate during the swollen period. The fitted values of the GLMMs are shown on the y-axis of panels a and $b$. The violin plots show the predicted probabilities (for a and b) or the raw rates (for c). Pairwise comparisons across female reproductive states and corresponding p-values are shown. 'ns': not significant $(\mathrm{p}>0.05) ; *: \mathrm{p}<0.05 ; * *: \mathrm{p}<0.01 ; * * *: \mathrm{p}<0.001$. 
Table 1: Male aggression in relation to female reproductive state. Significant p-values and confidence intervals that did not cross zero appear in bold. The significance of each variable was assessed using chi-square tests (Chisq), while the significance of each level of a categorical variable was evaluated against a reference level (noted 'Ref') according to whether their confidence intervals (CI) overlap or not.

\begin{tabular}{|c|c|c|c|c|c|}
\hline \multicolumn{6}{|c|}{ Response variable: Probability of receiving aggression from adult males $(0 / 1)$} \\
\hline Fixed Factor & Level & Estimate & CI $95 \%$ & Chisq & P-value \\
\hline \multirow[t]{6}{*}{ Reproductive State } & Swollen (Ref: Non-Swollen) & 0.463 & {$[0.186 ; 0.74]$} & 15.744 & 0.001 \\
\hline & Pregnant (Ref: Non-Swollen) & 0.066 & {$[-0.141 ; 0.272]$} & & \\
\hline & Lactating (Ref: Non-Swollen) & -0.075 & {$[-0.293 ; 0.142]$} & & \\
\hline & Swollen (Ref: Lactating) & 0.539 & {$[0.266 ; 0.812]$} & & \\
\hline & Pregnant (Ref: Lactating) & 0.141 & {$[-0.054 ; 0.337]^{-}$} & & \\
\hline & Swollen (Ref: Pregnant) & 0.398 & {$[0.137 ; 0.659]$} & & \\
\hline \multirow[t]{2}{*}{ Female Rank } & Low Rank (Ref: High Rank) & -0.740 & {$[-1.015 ;-0.466]$} & 29.450 & $<0.001$ \\
\hline & Medium Rank (Ref: High Rank) & -0.515 & {$[-0.878 ;-0.153]$} & & \\
\hline Female Parity & Parous (Ref: Nulliparous) & 0.040 & {$[-0.350 ; 0.429]$} & 0.040 & 0.842 \\
\hline Group Sex Ratio & & -0.038 & {$[-0.083 ; 0.007]$} & 2.697 & 0.101 \\
\hline
\end{tabular}

Table 2: Injuries in relation to female reproductive state. Significant p-values and confidence intervals that did not cross zero appear in bold. The significance of each variable was assessed using chi-square tests (Chisq), while the significance of each level of a categorical variable was evaluated against a reference level (noted 'Ref') according to whether their confidence intervals (CI) overlap or not.

\begin{tabular}{|c|c|c|c|c|c|}
\hline \multicolumn{6}{|c|}{ Response variable: Probability of having an injury $(0 / 1)$} \\
\hline Fixed Factor & Level & Estimate & CI $95 \%$ & Chisq & P-value \\
\hline \multirow[t]{6}{*}{ Reproductive State } & Swollen (Ref: Non-Swollen) & 1.183 & {$[0.579 ; 1.787]$} & 34.535 & $<0.001$ \\
\hline & Pregnant (Ref: Non-Swollen) & -0.452 & {$[-1.026 ; 0.123]$} & & \\
\hline & Lactating (Ref: Non-Swollen) & -0.507 & {$[-1.076 ; 0.061]$} & & \\
\hline & Swollen (Ref: Lactating) & 1.656 & {$[1.013 ; 2.299]$} & & \\
\hline & Pregnant (Ref: Lactating) & 0.100 & {$[-0.503 ; 0.704]$} & & \\
\hline & Swollen (Ref: Pregnant) & 1.556 & {$[0.943 ; 2.169]$} & & \\
\hline \multirow[t]{2}{*}{ Female Rank } & Low Rank (Ref: High Rank) & 0.203 & {$[-0.396 ; 0.802]$} & 2.812 & 0.245 \\
\hline & Medium Rank (Ref: High Rank) & -0.418 & {$[-1.146 ; 0.310]$} & & \\
\hline Female Parity & Parous (Ref: Nulliparous) & 0.132 & {$[-0.826 ; 1.090]$} & 0.073 & 0.787 \\
\hline Group Sex Ratio & & -0.013 & {$[-0.109 ; 0.083]$} & 0.071 & 0.789 \\
\hline
\end{tabular}


Table 3: Male aggression and mating success. Probability of copulation of an heterosexual dyad during a female's swollen period in relation to the rate of male aggression received before that swollen period. Significant p-values and confidence intervals that did not cross zero appear in bold. The significance of each variable was assessed using chi-square tests (Chisq), while the significance of each level of a categorical variable was evaluated against a reference level (noted 'Ref') according to whether their confidence intervals (CI) overlap or not.

\begin{tabular}{|c|c|c|c|c|c|}
\hline \multicolumn{6}{|c|}{ Response variable: Mating during the swollen period $(0 / 1)$} \\
\hline Fixed Factor & Level & Estimate & CI $95 \%$ & Chisq & $\mathrm{P}$-value \\
\hline Aggression Rate & & 1.622 & {$[0.174 ; 3.069]$} & 4.823 & 0.028 \\
\hline Male Rank & Alpha (Ref: Non-alpha) & 1.229 & {$[0.483 ; 1.976]$} & 10.420 & 0.001 \\
\hline Female Rank & Low Rank (Ref: High Rank) & 0.646 & {$[-0.206 ; 1.498]$} & 2.464 & 0.292 \\
\hline & Medium Rank (Ref: High Rank) & 0.665 & {$[-0.657 ; 1.986]$} & & \\
\hline Female Parity & Parous (Ref: Nulliparous) & -0.461 & {$[-2.797 ; 1.876]$} & 0.149 & 0.699 \\
\hline Operational Sex Ratio & & -0.001 & {$[-0.514 ; 0.511]$} & 0.000 & 0.996 \\
\hline
\end{tabular}

